\title{
Metodología alternativa para la aplicación del aprendizaje servicio en la Educación Básica
}

\author{
Alexandro Escudero Nahón, Azucena Ochoa Cervantes \\ Universidad Autónoma de Querétaro. México
}

\begin{abstract}
Resumen
Las experiencias de aprendizaje servicio son estrategias educativas que permiten al alumnado salir de los salones de clase y colaborar en la solución de un problema sentido de la comunidad. Este servicio a la comunidad siempre está vinculado al aprendizaje de un contenido formal del currículum, pero sus resultados generalmente exceden esas expectativas de aprendizaje. Esta investigación en proceso encontró que la aplicación de la metodología habitual del aprendizaje servicio encuentra varias dificultades legales, administrativas, escolares y conceptuales, cuando se intenta aplicar en la educación básica del estado de Querétaro, México. Entre todas estas dificultades destaca el hecho de que el alumnado no puede salir de las instalaciones escolares. Lo anterior derivó en el diseño de una estrategia alternativa que implica una reformulación conceptual y una reformulación metodológica del aprendizaje servicio. Conceptual, porque es necesario considerar a la comunidad escolar, en sí misma, una comunidad con necesidades sociales; metodológica, porque se adapta un procedimiento para el diseño de unidades didácticas por competencias (que se lleva a cabo dentro de la escuela), para mejorar la convivencia escolar
\end{abstract}

\section{Palabras clave}

Aprendizaje servicio, práctica educativa, educación básica.

Fecha de recepción: 26/V/2015

Fecha de aceptación: 14/IV/2016 


\title{
Alternative methodology for the implementation of service learning in Basic Education
}

\begin{abstract}
Service Learning experiences are educational strategies that encourage students to collaborate on solving sensitive problems of their community by leaving the classroom. This community service is always linked to learning a formal content of the curriculum, but the results generally exceed those expectations on learning. This ongoing research found that the implementation of the usual methodology of Service Learning faces several legal, administrative, educational and conceptual difficulties when attempting to develope a compulsary education experience in the state of Queretaro, Mexico. Among all these difficulties, it is relevant the fact that students can not leave the school facilities. Thus, the research group was led to the design of an alternative strategy, taking into account the difficulty of making a community service by usual procedures. The main feature of this proposed strategy involves a conceptual reformulation and a methodological reformulation of Service Learning. A conceptual reformulation, because it is necessary to consider the school community, itself, as a community with social needs; A methodological reformulation, because it is proposed an adaptation of the design process of a teaching unit based on competency, in order to improve school coexistence.
\end{abstract}

\section{Keywords}

Service learning, educative practice, basic education. 


\section{Introducción}

El aprendizaje servicio (ApS) es una estrategia educativa que combina procesos de aprendizaje y de servicio a la comunidad en un solo proyecto. El alumnado que participa en alguna experiencia de ApS tiene la oportunidad de colaborar en la solución de problemas considerados importantes por un grupo de personas de su comunidad $y$, al mismo tiempo, aprender nuevos valores y saberes para la participación social (Puig, 2007; 2009).

Es importante distinguir al ApS entre otras propuestas de participación escolar institucionalizadas porque comúnmente se le confunde con actividades extra escolares, participación comunitaria, voluntariados, prácticas culturales, visitas guiadas, etcétera. Para lograr lo anterior, cuatro cuadrantes formados por los ejes del aprendizaje y del servicio resultan útiles (ver figura 1).

El ApS, localizado en el cuadrante superior derecho, se diferenciaría de otras estrategias de participación social principalmente porque asume que mientras más servicio se ofrece a la comunidad, más se aprende. Así, el ApS no debería ser confundido con el voluntariado, el servicio social, las prácticas profesionales o las actividades extra clase, pues ninguna de ellas hace explícito su interés en aprender un contenido curricular a través de un servicio a la comunidad.

Figura 1: El aprendizaje servicio entre otras estrategias de participación social (adaptado de Tapia, 2000)

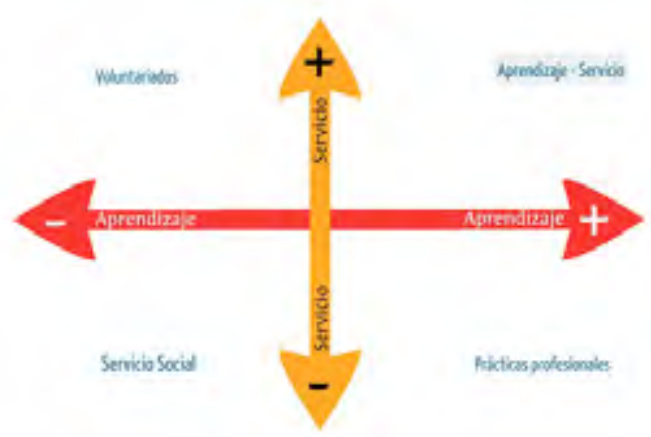

Lo anterior significa que el ApS intenta cerrar sistemáticamente la brecha entre la experiencia práctica de servicio a la comunidad y el proceso de aprendizaje (Paso Joven, 
2004). Logra lo anterior por dos vías:

1. Hace explícito el objetivo de aprendizaje de un contenido curricular

2. Transversaliza un proceso de reflexión sobre lo aprendido a lo largo de todo el proceso de servicio a la comunidad

El carácter no formalde esta estrategia se basa en el hecho de que el alumnado debe ir a colaborar en la solución de problemas de la comunidad, en la comunidad. Es decir, debe abandonar el salón de clases, las instalaciones escolares, y participar en y con la comunidad.

\section{El procedimiento del aprendizaje servicio}

Es ampliamente aceptado el hecho de que un proyecto de ApS debe contar con una serie de pasos flexibles, pero rigurosos (CLAYSS, 2009)

Tabla 1: pasos fundamentales del aprendizaje servicio

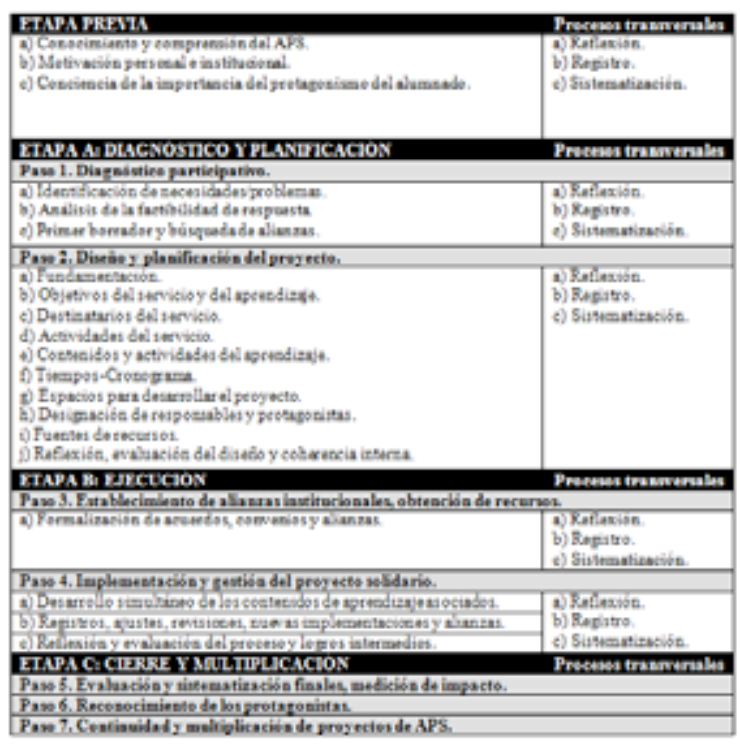

Esta serie de pasos a manera de guía resulta ser muy útil una vez que el ApS ya ha sido aceptado entre los principales actores de la estrategia, a saber: autoridades educativas, profesorado, alumnado, comunidad, y madres y padres de familia.

Sin embargo, esta investigación en proceso encontró que la aplicación de la metodología habitual del ApS encuentra varias dificultades legales, administrativas, escolares y conceptuales, cuando se intenta 
aplicar por primera vez en la educación básica del estado de Querétaro. Entre todas estas dificultades destaca un hecho fundamental: el alumnado no puede abandonar las instalaciones escolares por varios motivos.

A través de varias entrevistas en profundidad al grupo de investigadoras e investigadores que tienen por objetivo iniciar un programa piloto para aplicar el ApS en diversos niveles de educación básica, de la compilación de documentos que dan cuenta de las estrategias que se han tenido que realizar, y de entrevistas a autoridades educativas, se analizaron los desafíos conceptuales y metodológicos que implicaría aplicar por primera vez el ApS en la educación básica en Querétaro, México.

\section{Metodología de investigación}

Inspirado en el proceso de codificación en tres pasos de la metodología de la Teoría
Fundamentada, este estudio en proceso intenta identificar los principales motivos que impiden una aplicación habitual del ApS en la educación básica en la ciudad de Querétaro, México.

La Teoría Fundamentada es un procedimiento general de investigación eminentemente inductivo. Es especialmente útil para construir categorías analíticas basadas en datos. Su objetivo principal no es verificar o comprobar teoría, como sucede en la investigación hipotético-deductiva, sino intentar construir explicaciones fundamentadas en datos obtenidos en el campo de estudio.

La codificación en tres pasos implica el siguiente procedimiento (Strauss y Corbin, 2008):

1. Codificación abierta. El primer paso de la codificación hace referencia a "abrir el texto". Es decir, obtener información y generar datos relevantes para dotarles de una categoría. El 
procedimiento de obtención de información admite diversas técnicas y procedimientos. Aunque generalmente la información se obtiene a través de entrevistas en profundidad, el análisis de documentos también es útil y deseable.

2. Codificación axial. Esta fase hace referencia a identificar una categoría central entre todas las categorías generadas (identificar un eje). Posteriormente se ordenan alrededor de la categoría axial, las categorías periféricas agrupadas como elementos de un proceso. Para lograr esto resultan útiles las "Familias de códigos" que ha propuesto Glaser (1978) en la literatura especializada sobre Teoría Fundamentada. Dichas familias son representaciones gráficas donde pueden ser "ordenadas" las categorías producidas en la codificación abierta. Este ordenamiento permite analizar el fenómeno social como un proceso, compuesto de varios elementos en relación.

3. Codificación teórica. El tercer y último procedimiento de codificación tiene por objetivo trascender la descripción detallada del fenómeno estudiado y construir explicaciones conceptuales, adaptar estrategias, considerar procedimientos alternativos, a manera de propuestas de acción (Gibson y Hartman, 2014).

En esta investigación fueron realizadas seis entrevistas en profundidad y analizados tres proyectos educativos con la intención de identificar los factores que dificultan la aplicación habitual de la metodología del ApS en la educación básica en Querétaro.

\section{Análisis de datos}

\subsection{Codificación abierta}

Se realizaron seis entrevistas en profundidad para conocer las 
dificultades que habían surgido al momento de aplicar la metodología del ApS en la educación básica. Las entrevistas tuvieron carácter anónimo, y fueron aplicadas a una profesora investigadora experta en el tema de convivencia escolar, a tres becarios del CONACYT, y a dos autoridades escolares de Querétaro. Una de las autoridades labora como director de una escuela de educación primaria; la otra autoridad, labora como director de una escuela de educación secundaria.

Los ámbitos que se exploraron y las categorías más relevantes que surgieron tras la codificación abierta se presentan en la tabla 2.

Destaca el hecho de que el ApS genera un interés positivo de inmediato porque su formulación es, en sí misma, original y útil. Sin embargo, las autoridades escolares y el profesorado requieren y solicitan que el proyecto de ApS se presente por escrito usando formatos convencionales, y que se adapte a los horarios y procesos educativos de las escuelas. Así lo relata una de las personas que intentó iniciar un proyecto de APS:

Entrevistador: ¿Qué fue lo que hicieron y qué dificultades encontraron?

Entrevistada: Lo que dijimos fue "queremos echar mano de la metodología de Aprendizaje Servicio, que implica... como el... poder vincular los contenidos académicos con algún servicio a la comunidad... como tratando de encontrar esta función social que tiene la escuela y poder aprender significativamente esto que se está viendo en las aulas. Y pues, de esta forma, también como resaltando el tema de la participación, que es donde estamos entrando más". Al director le gustó mucho y dijo "Sí, adelante, lo que ustedes necesiten $y$, pues vengan, ¿no?". $Y$ después dijo: "necesito que me describas, necesito que me traigas algo en concreto porque no entiendo qué quieres hacer ¿cuántas sesiones quieres?, ¿cuál es tu objetivo? ¿cuál es tu tema? Necesito que todas las ideas que traigas me las escribas..." Así que lo que hicimos Azucena y yo, fue sentarnos a poner fechas, que era lo que quería: cuánto tiempo vas a estar aquí y qué vas a hacer. Les pusimos una calendarización que igual te la puedo enseñar porque 
necesito saber si es factible lo que voy a hacer. Al final dijeron "iAh! bueno sí, pues te damos una hora a la semana, tal día vienes, adelante" pero...

Entrevistador: ¿Pero de qué manera salen a la comunidad?

Entrevistada: Todavía no, llevamos una sesión y media. Me dieron los lunes de 9 a 10 de la mañana. Yo empecé hace 8 días ya a trabajar directamente con los chavos. Era mi primera sesión como de acercamiento $y$ reconocimiento $y$ me empalmaron una actividad ahí. Ya no pude hacer nada.

Lo anterior demuestra que una de las resistencias más importantes ante el ApS es la dificultad de concederle tiempo a agentes externos a la escuela para que desarrollen el ApS. Sin embargo, también existe una dificultad administrativa: si el ApS no se institucionaliza como práctica escolar, puede ser erradicado en cuanto haya cambio de niveles directivos. Así lo expresó uno de los directores de educación primaria:

Entrevistador: Cómo director ¿qué necesita para aplicar ApS en esta escuela?
Entrevistado: Yo necesito nada más, conociendo, conociendo sus bases, su fundamento, darlo a conocer a la supervisión. Por lo siguiente: los directivos, yo tengo seis años aquí, sin embargo, en cualquier momento se vienen movimientos... que en un momento dado le convienen a uno... acercarse a otra escuela y se quedaría aquí $y, y$ esos proyectos no pueden quedarse truncos. Ni pueden ser por situación de visto bueno del director que está, sino que deben ser institucionalizados.

Lo anterior implica que las personas que pretenden aplicar ApS, siendo agentes externos a la escuela, requieren poseer cierto poder de convencimiento y habilidades de gestión para lograr que el proyecto de APS sea institucionalizado.

Si las dificultades antes expuestas, es decir, la convencionalización y la institucionalización del ApS, son de por sí eventualidades que distraen recursos y tiempo para iniciar un proyecto de este tipo, existen otras dos dificultades, una de las cuales parece ser insalvable: primero: la 
idea de que el profesorado trabajará más al aplicar una experiencia de APS y que al salir de la escuela se perderá tiempo de la planeación docente convencional, y segundo: la imposibilidad de que el alumnado salga de la escuela. Así lo relata una investigadora entrevistada al respecto:

Entrevistador: Entonces, para poder, por lo menos, registrar las dificultades a las que se enfrenta un grupo de personas que quiera hacer Aprendizaje - Servicio, el ortodoxo ¿tú ves varios niveles de dificultades o varios ámbitos de dificultades? O ambos: varios ámbitos con sus distintos niveles. $O$ crees que sólo hay una dificultad que, resolviéndola, puede empezar a abrir las otras.

Entrevistada: Tal vez hay como ámbitos... Yo pienso que el ámbito principal es la parte de... de la cuestión de salir de la escuela. Es algo como fundamental, que creo que obstaculiza. Otro ámbito es la sensibilidad o la sensibilización que merece la comunidad escolar, en el sentido de lo que te decía hace un rato. Es importante darnos cuenta de qué se debe mejorar o cómo sensibilizarnos hacia los problemas... si pensáramos que pusiéramos un aprendizaje servicio ortodoxo. Sensibilizarnos a los problemas, eso yo creo que es como una dificultad: vemos todo como que así es y no hay nada que mejorar, aquí nos tocó y ni modo, yo creo que ése sería otro ámbito importante. Un ámbito es el asunto de lo administrativo, de salir de la escuela, y otro ámbito es la sensibilización que requiere la comunidad para poner en marcha estas estrategias y un último ámbito que observo es 10 pedagógico, en el sentido de que esta estrategia implicaría necesariamente la interdisciplina o la integración. Por lo menos de contenidos. Y para los maestros en general les es muy complejo hacer esta integración de contenidos. Habría que también pensar en esa dimensión, en el ámbito pedagógico que tendríamos que, de alguna manera, enseñar a los profesores, no sé si es la palabra adecuada a hacer esta integración de contenidos. 


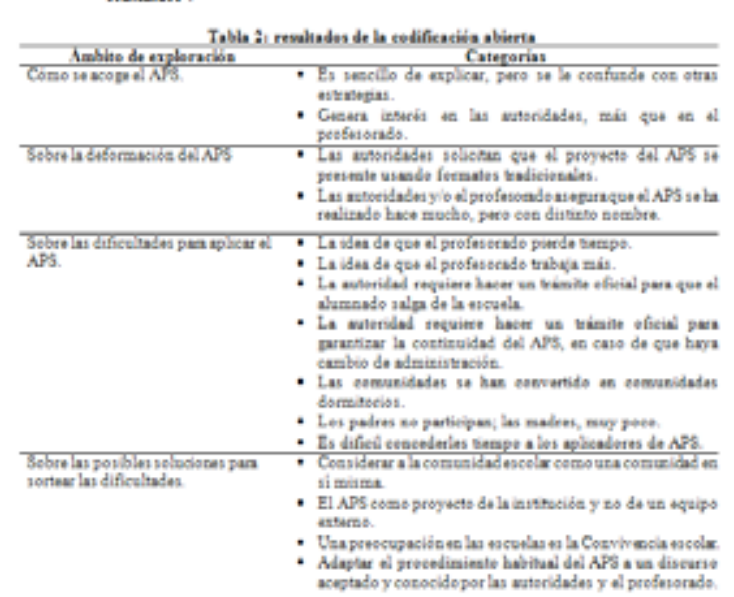

\subsection{Codificación axial}

La codificación axial tiene por objetivo seleccionar una categoría central (un eje) sobre la cual se relacionan las demas categorías agrupadas como elementos de un proceso. Este ejercicio se realiza usando una representación gráfica del fenómeno estudiado (ver figura 1). De acuerdo con la codificación axial de este estudio, se puede inferir que la metodología del ApS sí puede ser introducida en las escuelas que imparten educación básica, siempre y cuando se reelabore uno de los conceptos y uno de los procedimientos fundamentales de esa metodología: que el proyecto del ApS no exija al alumnado salir de la escuela, y que adopte un discurso conocido y un tema prioritario para la educación básica: el aprendizaje de competencias para la convivencia escolar.

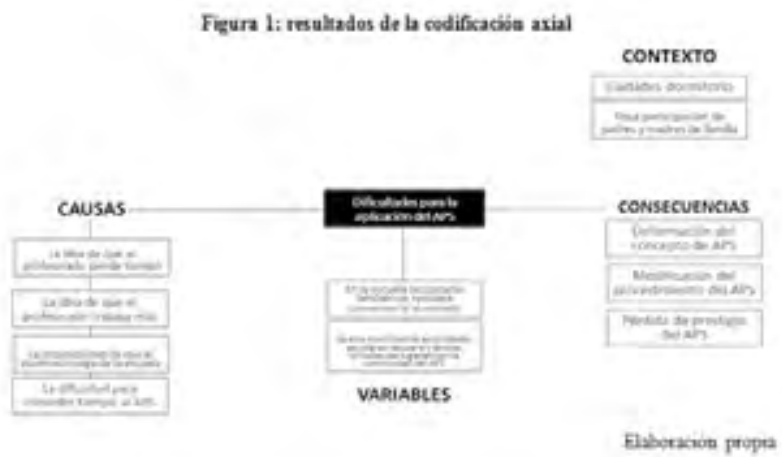




\section{Propuesta alternativa para la aplicación del ApS en la Educación Básica}

\subsection{La reelaboración conceptual}

La codificación teórica es, propiamente, la propuesta alternativa para aplicar ApS en la educación básica. Respecto a la reelaboración conceptual del ApS, que es la imposibilidad de que el alumnado salga de la escuela para colaborar en la resolución de un problema sentido de la comunidad, debería fomentarse la idea de que la comunidad escolar, en sí misma, es una comunidad que requiere la participación de su alumnado para resolver problemas comunes.

El Método de aprendizaje experiencial de Kolb (1984), es un procedimiento sencillo y adecuado para que el profesorado fomente escenarios de aprendizaje experiencial dentro de las aulas. Este procedimiento tiene la característica de iniciar con la inducción a una experiencia y terminar con una llamada a la acción. Se compone de cuatro fases que sí se pueden desarrollar dentro de la propia escuela:

1. Experiencia concreta. EI profesorado induce a que el alumnado experimente un problema en su escuela, como puede ser la falta de árboles, la suciedad en los muros, la poca convivencia entre el alumnado, etcétera. De acuerdo con las entrevistas realizadas, el tema de la convivencia escolar ha sido considerado, tanto por las autoridades escolares, como por la experta en educación entrevistada, un tema prioritario y susceptible de ser abordado con esta propuesta alternativa de ApS.

2. Observación reflexiva. EI profesorado aplica un proceso puntual de reflexión. A diferencia del ApS, que requiere un proceso de transversalización reflexiva complejo, la propuesta del aprendizaje experiencial es muy puntual. En esta etapa el 
profesorado induce a que el alumnado vea las desventajas que implica no resolver cierto problema en la escuela. Al discutir en grupo por qué es importante resolver problemas en la escuela (el problema de la convivencia escolar, por ejemplo), ya se ponen en marcha una serie de aprendizajes no formales.

3. Conceptualización abstracta. En esta fase, el profesorado provoca la articulación entre el contenido curricular que el alumnado debe aprender y la reflexión sobre la manera de resolver el problema en cuestión. En esta propuesta se pondrá especial atención al tema de la convivencia escolar, que es un ámbito de estudio que goza de amplia aceptación entre las autoridades escolares de Querétaro, México.

4. Experimentación activa. Finalmente, el profesorado induce a la acción. Puesto que es una propuesta surgida en su asignatura, el profesorado puede concederle tiempo suficiente para que el alumnado realice gestiones dentro de la escuela, se reúna con otros grupos de alumnas y alumnos, intente resolver un problema y se vea obligado a aprender ciertos conceptos para concluir exitosamente el proyecto.

\subsection{La reelaboración metodológica}

Respecto a la reelaboración metodológica del ApS, que ha sido motivada porque existe una imposibilidad de aplicar un proceso complejo para que el alumnado sea consciente de qué aprendió y cómo lo aprendió, se propone la adaptación de la metodología de diseño de una secuencia didáctica (Tobón, 2013).

De acuerdo con la información obtenida en este estudio, el discurso sobre el aprendizaje basado en competencias está bien aceptado 
entre el profesorado y las autoridades de la educación básica. El equipo de aplicación del ApS (que es externo a la escuela) podría ingresar con más facilidad al aula si propone un procedimiento de actuación sencillo, y un instrumento de evaluación que considere los elementos de las competencias educativa (ver figura 3).

A diferencia del proceso complejo del ApS, el diseño de una secuencia didáctica constaría de seis fases, donde el proceso reflexivo (fundamental para el ApS), se realiza al final, puntualmente, pero sí se realiza.

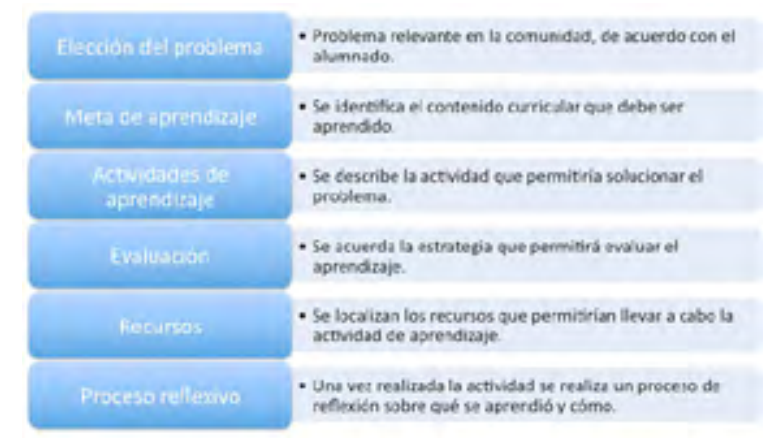

Figura 3: diseño de una secuencia didáctica. Elaboración propia

\section{Conclusiones}

La potencia del APS reside en que alumnado sale de los salones de clase y colabora en la solución de un problema sentido de la comunidad. Este servicio a la comunidad siempre está vinculado al aprendizaje de un contenido formal del currículum, pero se ha demostrado que sus resultados generalmente exceden esas expectativas de aprendizaje.

Cuando el autor la y la autora de este texto intentaron aplicar por primera vez la metodología del ApS en una escuela de educación primaria en la ciudad de Querétaro, México, aparecieron varias dificultades para llevar a cabo la experiencia. Las dificultades legales, administrativas, escolares y conceptuales para aplicar la metodología del ApS de manera habitual en Querétaro, México, ha impulsado una propuesta alternativa para desarrollar un proceso de aprendizaje de competencias para la mejora de la convivencia escolar.

Dicha propuesta se basa en dos 
reformulaciones: una conceptual y otra metodológica. La reformulación conceptual hace referencia a que la comunidad escolar puede ser considerada, en sí misma, como una comunidad con problemas que pueden ser resueltos con ayuda del alumnado. Esta reformulación es innovadora y original para el ApS. Para lograr que la comunidad escolar sea percibida como una comunidad en sí misma, se propone una adaptación d e I Método de aprendizaje experiencial de Kolb, que ha demostrado tener buenos resultados en la educación formal.

La reformulación metodológica se basa en una adaptación del proceso de reflexión sobre qué se aprende y cómo se aprende al colaborar en la solución de un problema de la comunidad (fundamental para el ApS). Dicha adaptación se realiza al adoptar la metodología para el diseño de una secuencia didáctica basada en el aprendizaje de competencias, con el objetivo de mejorar la convivencia escolar.
Hace falta más investigación empírica para saber si dicha propuesta tiene efectos deseables en las escuelas. Ambas reformulaciones permitiría que las personas que desean aplicar el ApS de manera habitual, tengan un primer contacto con un escenario escolar donde ha sido difícil implementar el ApS, pero donde sí existe interés manifiesto por avanzar en ese sentido. 


\section{Referencias bibliográficas}

CLAYSS ( 2009 ) Centro Latinoamericano de Aprendizaje y Servicio Solidario-Natura. Creer para Ver. Manual para docentes y estudiantes solidarios. Buenos Aires, 2009.

Gibson, B., y Hartman, J. (2014). Rediscovering grounded theory. Beverly Hills: Sage.

Glaser, B. (1978). Theoretical sensitivity: advances in the methodology of grounded theory. Mill Valley: Sociology Press.

Kolb, D. (1984). Experiential Learning: Experience as the Source of Learning and Development. Michigan: Prentice-Hall.

Paso Joven (2004). Participación Solidaria para América Latina. Manual de formación de formadores en aprendizaje-servicio y servicio juvenil. BID-SES-CLAYSS-ALIANZA ONGCEBOFIL.

Puig, J. M. (2007). Aprendizaje
Servicio. Educar para la ciudadanía. Barcelona: Octaedro.

Puig, J. M. (coord.) (2009). Aprendizaje Servicio (ApS). Educación y compromiso cívico. (Crítica y fundamentos, 26) Barcelona: Graó.

Strauss, A., y Corbin, J. (2008). Basics of qualitative research: techniques and procedures for developing grounded theory (3a. ed.). Thousand Oaks, California [etc.]: Sage.

Tapia, M. N. (2000). Aprendizaje y servicio solidario en el sistema educativo y las organizaciones juveniles. Buenos Aires: Editorial Ciudad Nueva.

Tobón, S. ( 2013 ). Trabajo colaborativo e interaprendizaje. México: CIFE.

El grupo de investigadoras e investigadores está conformado por Azucena Ochoa Cervantes, Coordinadora de la Maestría en Educación para la Ciudadanía de la Universidad Autónoma de Querétaro, Alexandro Escudero, profesor en dicho programa educativo, y Josué Castro, Luis Pérez, y Laura Morales, becarios y becaria CONACYT en el mismo programa educativo. 
Los proyectos educativos analizados son autoría de Laura Lecona, Luis Pérez y Josué Castro, estudiantes de la Maestría en Educación para la
Ciudadanía, que coordina la Dra. Azucena Ochoa Cervantes en la Facultad de Psicología de la Universidad Autónoma de Querétaro.

Escudero, A.; Ochoa, A. (2016). Metodología alternativa para la aplicación del aprendizaje servicio en la Educación Básica. RIDAS, Revista Iberoamericana de Aprendizaje y Servicio, 2, 95-110.

DOI10.1344/RIDAS2016.2.5 